\title{
Retrograde TrkAIII transport from ERGIC to ER: a re-localisation mechanism for oncogenic activity
}

\author{
Antonietta Rosella Farina ${ }^{1}$, Lucia Cappabianca ${ }^{1}$, Pierdomenico Ruggeri ${ }^{1,2}$, Luciana \\ Gneo $^{1}$, Rita Maccarone ${ }^{1}$ and Andrew Reay Mackay ${ }^{1}$ \\ ${ }^{1}$ Department of Applied Clinical and Biotechnological Sciences, University of L'Aquila, L'Aquila, Italy \\ 2 Department of Medical-Surgical Science and Biotechnology, University of Rome "La Sapienza", Latina, Italy \\ Correspondence to: Andrew Reay Mackay, email: andrewreay.mackay@univaq.it \\ Keywords: TrkAlll oncoprotein, retrograde transport, activation mechanism, neuroblastoma, endoplasmic reticulum intermediate \\ compartment \\ Received: July 31, 2015 \\ Accepted: September 12, 2015 \\ Published: September 22, 2015
}

This is an open-access article distributed under the terms of the Creative Commons Attribution License, which permits unrestricted use, distribution, and reproduction in any medium, provided the original author and source are credited.

\section{ABSTRACT}

In human SH-SY5Y neuroblastoma (NB) cells, nascent immature N-glycosylated $110 k$ Da TrkA moves rapidly from the endoplasmic reticulum (ER) to the Golgi Network (GN), where it matures into the $140 \mathrm{kDa}$ receptor prior to being transported to the cell surface, creating GN and cell surface pools of inactive receptor maintained below the spontaneous activation threshold by a full compliment of inhibitory domains and endogenous PTPases. In contrast, the oncogenic alternative TrkAIII splice variant is not expressed at the cell surface but re-localises to intracellular membranes, within which it exhibits spontaneous ERGIC/COPI-associated activation and oncogenic Akt signalling. In this study, we characterise the mechanism responsible for TrkAIII re-localisation. Spontaneous TrkAIII activation, facilitated by D4 IG-like domain and $\mathrm{N}$-glycosylation site omission, increases spontaneous activation potential by altering intracellular trafficking, inhibiting cell surface expression and eliminating an important inhibitory domain. TrkAIII, spontaneously activated within the permissive ERGIC/COPI compartment, rather than moving in an anterograde direction to the GN exhibits retrograde transport back to the $E R$, where it is inactivated. This setsup self-perpetuating TrkAIII re-cycling between the ERGIC and ER, that ensures continual accumulation above the spontaneous activation threshold of the ERGIC/ COPI compartment. This is reversed by TrkA tyrosine kinase inhibitors, which promote anterograde transport of inactivated TrkAIII to the GN, resulting in GN-associated TrkAIII maturation to a $120 \mathrm{kDa}$ species that is degraded at the proteasome.

\section{INTRODUCTION}

The developmental and stress-regulated alternative TrkAIII splice variant of the NGF receptor tropomyosinrelated kinase, TrkA, is expressed by advanced stage human neuroblastomas (NBs), associates with poor prognosis and post therapeutic relapse in unfavourable high TrkA expressing tumours and exhibits oncogenic activity in NB models [1-6]. TrkAIII oncogenic activity is caused by exon 6/7 skipping, which results in the omission of the extracellular D4 IG-like domain and several $\mathrm{N}$-glycosylation sites, important for optimising intracellular trafficking, correct cell surface localisation, ligand binding and preventing spontaneous ligandindependent receptor activation [1, 7-9]. As a consequence and in contrast to TrkA, TrkAIII is not expressed at the cell surface but re-localises to intracellular membranes, within which it exhibits spontaneous ligand-independent activation, leading to oncogenic signalling through Akt but not Ras from this alternative location. Signalling from TrkAIII differs to classical signalling from cell surface ligand-activated TrkA through Ras/MAPK, suggesting that the re-localisation of TrkAIII is fundamental for it's oncogenic activity [1]. This hypothesis is supported by recent reports that the oncogenic activity of several receptor tyrosine kinase (RTK) oncogenes depends 
upon their re-localisation [10]. Mutation-activated FLT3 and c-KIT oncogenes, important in haematological malignancies, are retained within the ER, exhibit ERassociated spontaneous activation and oncogenic signalling through Akt/STAT5/PIM [11-12]. The Trk-T3 oncogene, involved in thyroid cancer, re-localises to ER exit sites (ERES), exhibits spontaneous activation within ERES-associated COPII vesicles and induces oncogenic signalling from this alternative location through Akt but not Ras, similar to that reported for the TrkAIII oncoprotein $[1,13]$.

The re-localisation of FLT3-ITD and c-Kit-ITD to the ER results from duplication of the internal tandem juxta-membrane domains, which impairs ER exit and facilitates spontaneous activation by compromising an important receptor inhibitory domain $[11,12]$. The relocalisation of Trk-T3 to the ERES/COPII compartment results from TrkA extracellular domain substitution with a chimeric Trk-fused gene (TFG) sequences that regulate COPII vesicle formation at ERES, with the elimination of important TrkA extracellular domain sequences further facilitating spontaneous activation [13]. Whatever mechanisms result in RTK oncogene re-localisation, all induce oncogenic signalling from an alternative substrate context $[1,10-13]$.

Interestingly, the mere overexpression of TrkA in some cell types results in spontaneous intracellular activation and altered signalling. In human SV40immortalised 293 kidney cells, TrkA overexpression results in spontaneous activation within the ERGIC, which causes GN disruption and inhibits further GNassociated TrkA maturation. This augments intracellular accumulation, facilitates spontaneous activation and results in altered signalling through Akt but not Ras from this alternative site, also similar to that reported for the TrkAIII oncoprotein $[1,14]$. This cell-specific effect most likely reflects slower rates of intracellular TrkA trafficking, maturation and cell surface translocation, leading to aberrant accumulation and spontaneous activation within the ERGIC, perpetuated by GN disruption [14].

In pursuit of novel therapeutic strategies to inhibit RTK oncogenic activity, therefore, it is not only important to characterise the mechanisms responsible for oncogenic RTK activation and the oncogenic signalling pathways activated but also to identify the specific sites to which RTK oncogenes re-localise, and the mechanisms that cause re-localization and facilitate spontaneous activation. In the present study, we report a novel mechanism to explain the re-localisation of the TrkAIII oncoprotein in human NB cells. This mechanism is characterised by activationdependent retrograde TrkAIII recycling from the ERGIC back to the ER, which reduces anterograde TrkAIII transport to the GN, impedes further GN-associated TrkAIII maturation and sets-up self-perpetuating TrkAIII accumulation above the threshold for spontaneous activation within the ERGIC/COPI compartment, resulting in oncogenic Akt signalling from this alternative location. This mechanism is prevented by TrkA inhibitors, which promote anterograde trafficking of inactivated TrkAIII from the ERGIC to the GN, resulting in GN-associated maturation of TrkAIII to a novel $120 \mathrm{kDa}$ species that fails to reach the cell surface and is degraded at the proteasome.

\section{RESULTS}

\section{TrkA localises to two distinct pools of inactive receptors in SH-SY5Y cells}

We have previously reported [3] and confirm here (Figure 1A) that TrkA trafficking from ER to GN and then to the cell surface in SH-SY5Y NB cells occurs without spontaneous activation and results in two distinct steadystate pools of inactive TrkA receptors at the cell surface and within the GN, the latter overlapping closely with the GN marker GM130 [3]. Treatment of TrkA SH-SY5Y cells with the PTPase inhibitor sodium orthovanadate (Vanadate, $0.1 \mathrm{mM}$ for 3 hours) induced tyrosine phosphorylation of cell surface but not GN-associated $140 \mathrm{kDa}$ TrkA, implicating endogenous PTPases in preventing spontaneous activation of cell surface $140 \mathrm{kDa}$ TrkA but not GN-associated TrkA (Figure 1A, 3 hours treatment and Figure 1B, 3 and 6 hours treatment).

\section{CEP-701 alters the localisation of intracellular immature N-glycosylated 100kDa TrkAIII}

The N-glycosylation status of $100 \mathrm{kDa}$ TrkAIII was compared to that of $110 \mathrm{kDa}$ and $140 \mathrm{kDa}$ TrkA following overnight treatment with the N-glycosylation inhibitor tunicamycin $(5 \mu \mathrm{g} / \mathrm{ml})$ [15]. Tunicamycin induced expression of $80 \mathrm{kDa}$ TrkA and $70 \mathrm{kDa}$ TrkAIII core proteins (Figure 2A), confirming an N-glycosylated status for $100 \mathrm{kDa}$ TrkAIII, $110 \mathrm{kDa}$ and $140 \mathrm{kDa}$ TrkA proteins (Figure 2A). Incubation of $100 \mathrm{kDa}$ TrkAIII immunoprecipitates with endoglycosidase $\mathrm{H}$ (250U per reaction) resulted in TrkAIII degradation to $80-85 \mathrm{kDa}$ fragments, confirming an immature N-glycan status for 100kDa TrkAIII (Figure 2B) [16].

The steady state intracellular distribution of TrkA and TrkAIII was compared in density-gradient ultracentrifugation purified membrane fraction prepared under untreated (control) and CEP-701-treated (100nM for 16 hours) conditions (Figure 3). In TrkA SH-SY5Y cells, $76.6+6.9 \%$ of immature $110 \mathrm{kDa}$ TrkA was ERassociated (calnexin positive fractions 5 and 6 ), $7.2+0.9 \%$ COP-associated (COPI/II positive fractions 3 and 4) and $16.2+1.5 \%$ GN-associated (TG46/GM130 positive fractions 1 and 2) (Figure 3A I-II), whereas $1.2+0.1 \%$ of mature $140 \mathrm{kDa}$ TrkA was ER-associated (fractions 5 and 6), 34.9+2.4\% COP-associated (fractions 3 and 4) and 

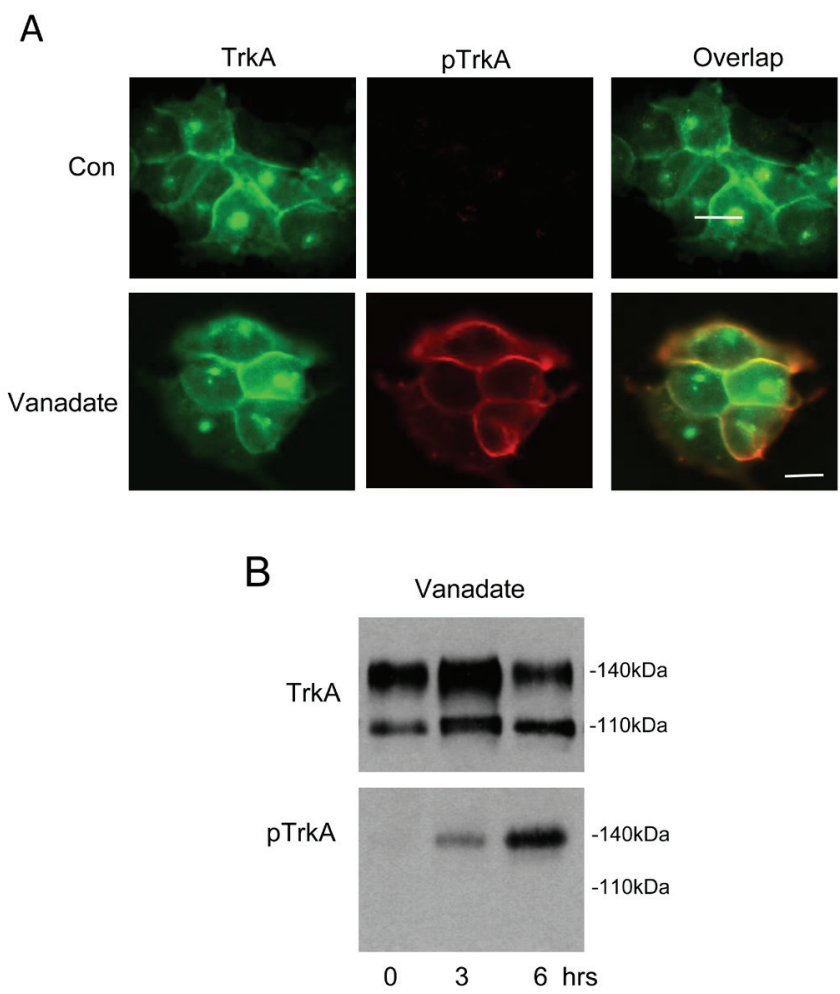

Figure 1: Cell surface but not GN-associated TrkA is activated by Sodium Orthovanadate. A. Indirect IF demonstrating overlap (yellow, bottom right panel) in surface and GN-associated TrkA (green, top and bottom left panels) and phosphorylated TrkA (pTrkA: red, top and bottom middle panels) in untreated (Con) and sodium orthovanadate-treated (Vanadate; $0.1 \mathrm{mM}$ for 3 hours) TrkA SH-SY5Y cells (bars $=10 \mu \mathrm{m})$. B. Western blot demonstrating the effect of sodium orthovanadate (vanadate; $0.1 \mathrm{mM}$ for 3 and 6 hours) on total (TrkA) and tyrosine phosphorylated TrkA (pTrkA) levels.
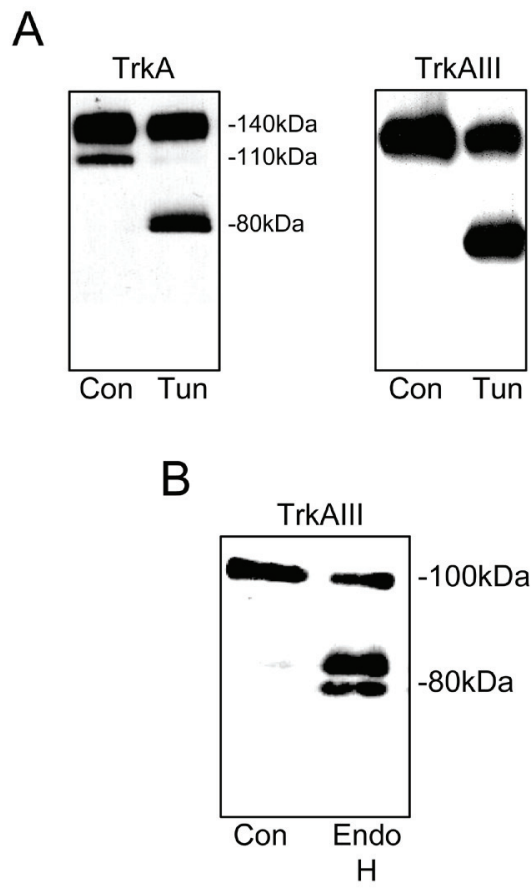

Figure 2: 100kDa TrkAIII is an immature N-glycosylated receptor. Western blots demonstrating the effect of tunicamycin (Tun, $0.1 \mathrm{mM}$ for 16 hours) on the molecular size of A) TrkA and TrkAIII, in total TrkA SH-SY5Y and TrkAIII SH-SY5Y cell extracts (30 $\mu g$ ). B) Western blot demonstrating degradation of immunoprecipitated 100kDa TrkAIII (Con) by Endoglycosidase H (Endo H). 
$63.8+3.8 \% \mathrm{GN}$-associated (fractions 1 and 2) (Figure 3A I-II), confirming GN-associated $140 \mathrm{kDa}$ TrkA maturation. CEP-701 (100nM) did not significantly alter the steady state distribution of either $110 \mathrm{kDa}$ or $140 \mathrm{kDa}$ TrkA (Figure 3B I-II). TrkA tyrosine phosphorylation was not detected (Figure 3A).

The steady state distribution of immature $100 \mathrm{kDa}$ TrkAIII differed to that of immature TrkA and was $38.9+3.3 \%$ ER-associated (fractions 5 and 6), 33.5+2.8\% COP-associated (fractions 3 and 4), 7+8.3\% associated
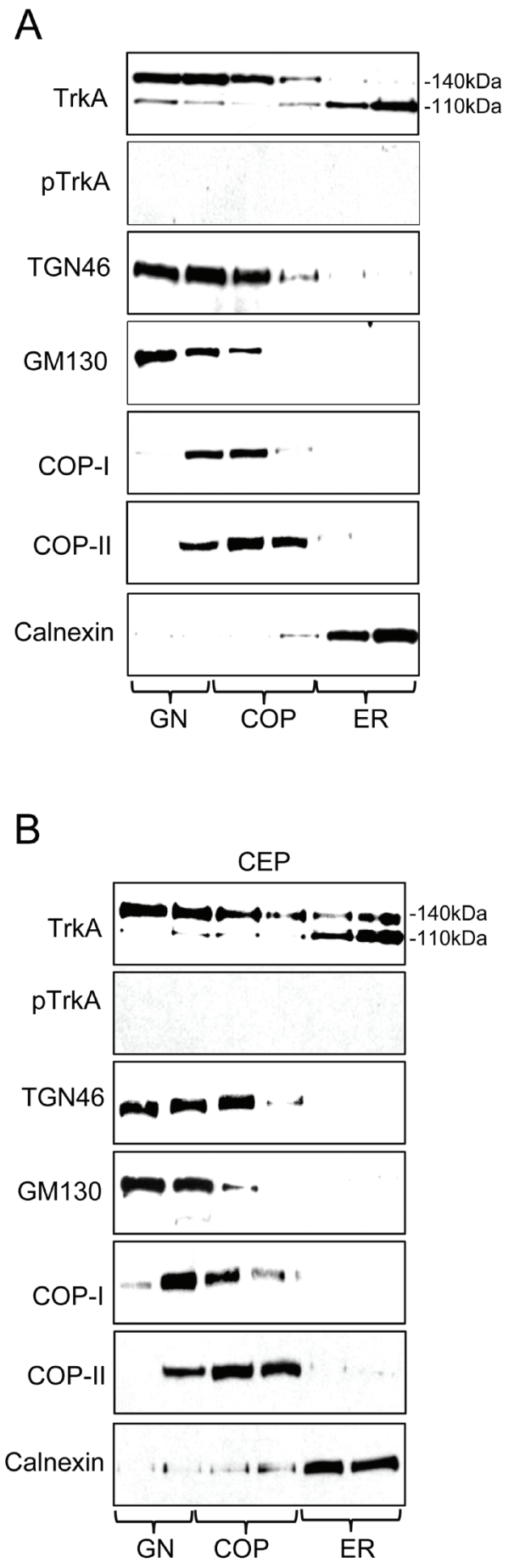

with GN-COP-negative fraction 1 and $23.5+2.7 \%$ associated with GN-COP-positive fraction 2 (Figure $3 \mathrm{C}$ I-II), indicating that immature $100 \mathrm{kDa}$ TrkAIII does not accumulate in COP-negative, TGN46/GM130 positive GN membranes. Spontaneous TrkAIII Tyrosine phosphorylation was detected primarily in COPI/II positive membranes $(76.5+7 \%$ in COP positive fractions 3 and 4), whereas only $5.8+0.5 \%$ was ER-associated (fractions 5 and 6 ) and $17.5+2.1 \%$ GN-associated (fractions 1 and 2) (Figure 3C I-II). No other TrkAIII
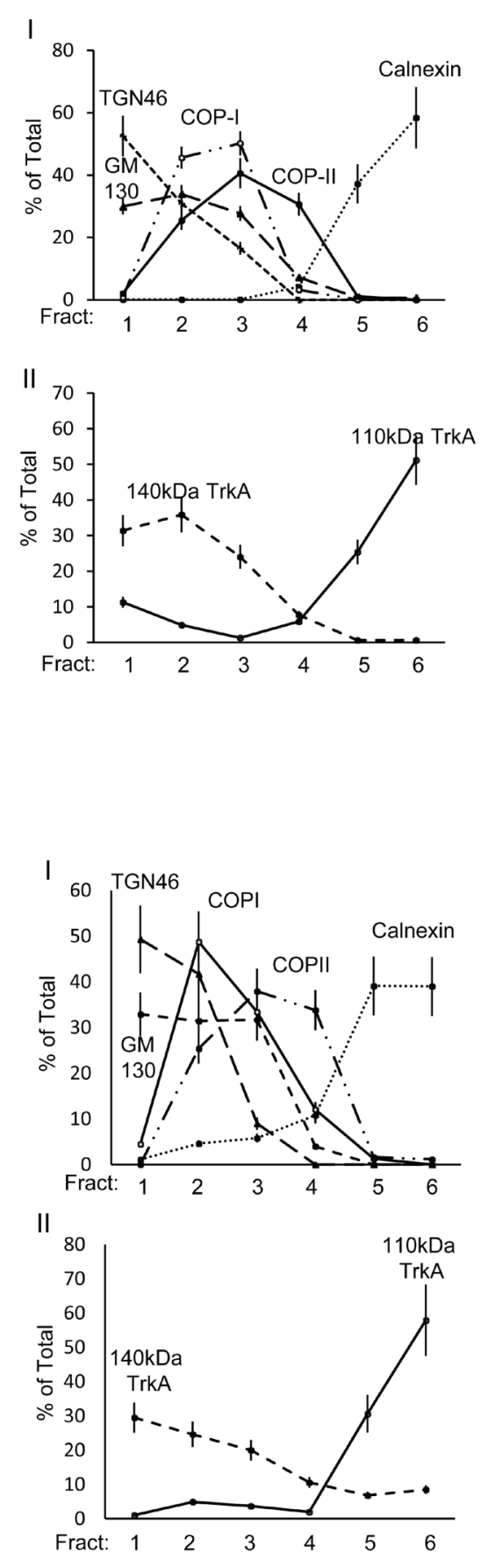

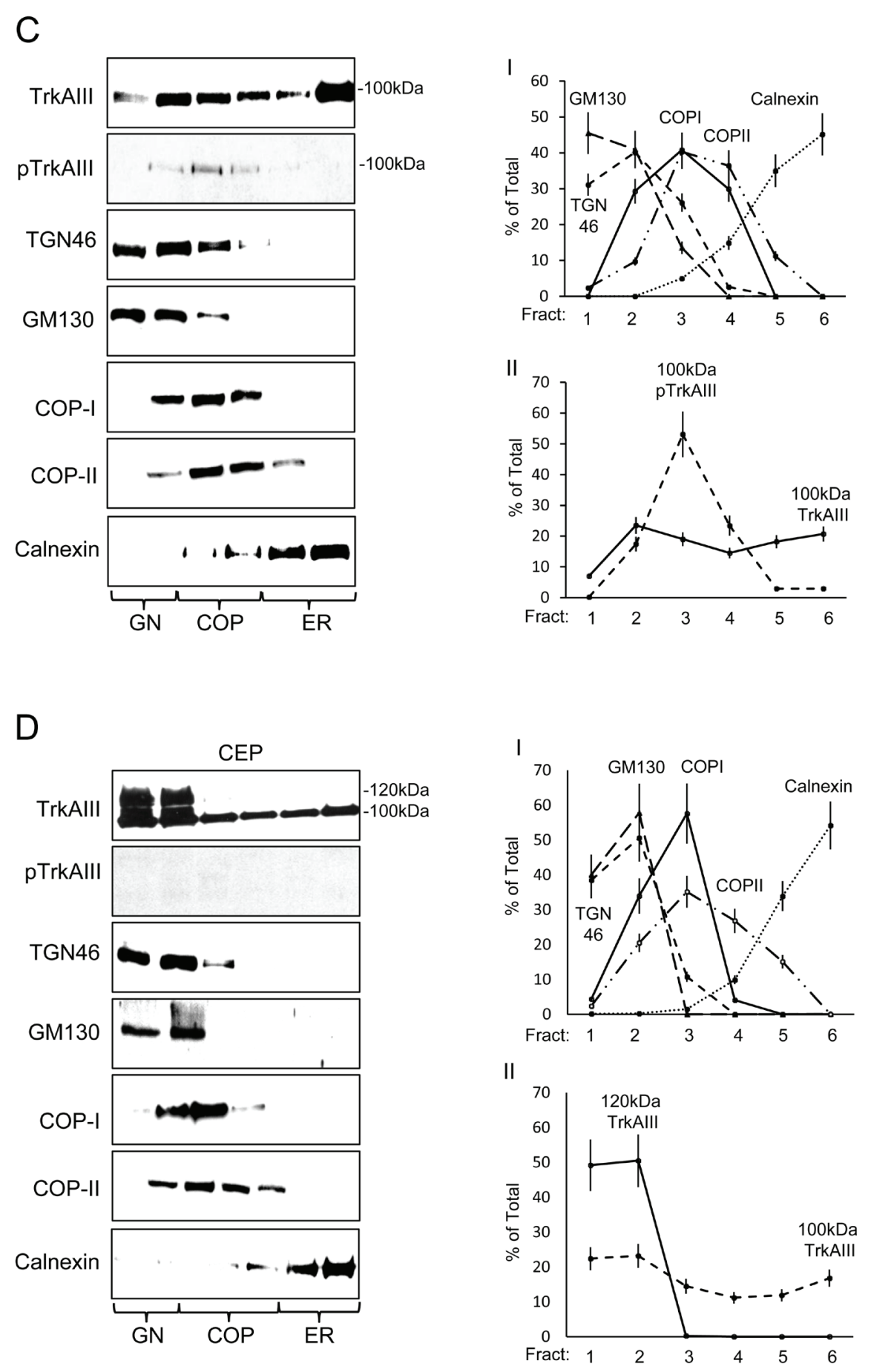

Figure 3: TrkAIII differs to TrkA in steady-state intracellular distribution and activation. Representative Western blots plus line graphs demonstrating steady state levels of: 140kDa and 110kDa TrkA; Y674/675 phosphorylated TrkA (pTrkA); 120kDa and 100kDa TrkAIII; Y674/675 phosphorylated TrkAIII (pTrkAIII); TGN46; GM130; COP-I, COP-II and calnexin in density-gradient ultracentrifugation purified intracellular membranes fractions from: A. untreated TrkA SH-SY5Y cells; B. CEP-701-treated (CEP; 100nM for 12 hours) TrkA SH-SY5Y cells; C. untreated TrkAIII SH-SY5Y cells; and D. CEP-701-treated (CEP; 100nM for 12 hours) TrkAIII SH-SY5Y cells. Line graphs depict GM130, TGN46, COP-I, COP-II and calnexin levels in: AI) untreated TrkA SH-SY5Y cells; BI) CEP-701-treated (CEP; 100nM for 12 hours) TrkA SH-SY5Y cells; CI) untreated TrkAIII SH-SY5Y cells; and DI) CEP-701-treated (CEP; 100nM for 12 hours) TrkAIII SH-SY5Y cells. Levels of 110kDa and 140kDa TrkA are displayed in AII) for untreated TrkA SH-SY5Y cells and in BII) for CEP-701-treated TrkA SH-SY5Y cells (CEP). Total and tyrosine phosphorylated 100kDa TrkAIII levels are displayed in CII) for untreated TrkAIII SH-SY5Y cells and levels of 100kDa and 120kDa TrkAIII displayed in DII) for CEP-701-treated TrkAIII SH-SY5Y cells (CEP) (presented as mean \pm s.d. \% total, from densitometric analysis of three independent experiments performed in duplicate). 
species were detected.

CEP-701 inhibited TrkAIII tyrosine phosphorylation and significantly increased localization of 100kDa TrkAIII to $45.6 \pm 4.5 \%$ in GN membranes (fractions 1 and 2 ; versus untreated controls $P=0.019, \mathrm{df}=6$ ) and significantly reduced TrkAIII in COP-membranes to $25.7 \pm 2.6 \%$ (fractions 3 and 4; versus untreated controls $\mathrm{P}=0.0065$, $\mathrm{df}=6$ ) and in ER-membranes to $28.7 \pm 2.9 \%$ (fractions 5 and 6 versus untreated controls $P=0.0035, \mathrm{df}=6$ ), indicating increased transport to the GN (Figure 3D I-II). CEP-701 also induced the expression of an additional $120 \mathrm{kDa}$ TrkAIII species, localized $51 \pm 7.6 \%$ to COP negative TGN46/GM130 positive GN membrane fraction 1 and $49 \pm 7.4 \%$ to TGN46/GM130 positive-COP positive fraction 2 (Figure 3D I-II). As a consequence, total TrkAIII $(100 \mathrm{kDa}$ plus $120 \mathrm{kDa})$ levels were significantly increased to $34 \pm 1.7 \%$ (versus untreated controls $P<0.0001$, $\mathrm{df}=$ 6) in COP negative TGN46/GM130 positive GN fraction 1 , to $33.7 \pm 5 \%$ in TGN46/GM130 positive COP positive membrane fraction 2 (versus untreated controls $P=$ 0.0013 , $\mathrm{df}=6)$ and significantly reduced to $15.6 \pm 1.7 \%$ in COP-membranes (fractions 3 and 4; versus untreated controls $P<0.0001, n=6$ ) and $16.7 \pm 1.8 \%$ (fractions 5 and 6; versus untreated controls $P<0.0001, \mathrm{df}=6$ ) in ER-membranes (Figure 3D I-II). Therefore, CEP-701 promotes TrkAIII transport from ER to GN and induces GN-associated 120kDa TrkAIII maturation (Figure 3D).

\section{Activated TrkAIII recycles back to the ER}

Metabolically labelling was used to further interrogate TrkA and TrkAIII trafficking over 60 minutes in density gradient ultracentrifugation separated membranes.

In TrkA SH-SY5Y cells (Figure 4A) at 15 minutes post-labelling, $79.2 \pm 10.8 \%$ of immature $110 \mathrm{kDa}$ TrkA was ER-associated (fractions 5 and 6), 26.9 $\pm 2.3 \%$ COPassociated (fractions 3 and 4), 5.9 $\pm 0.6 \% \mathrm{GN}$-associated (fractions 1 and 2) and low-level $140 \mathrm{kDa}$ TrkA was detected exclusively in GN fractions 1 and 2. Total TrkA (110kDa plus $140 \mathrm{kDa}$ ) was, therefore, $58.7 \pm 4.6 \%$ ERassociated, $23.5 \pm 1.8 \%$ COP-associated and $17.8 \pm 1.5 \%$ GN-associated. By 30 minutes, ER-associated $110 \mathrm{kDa}$ TrkA had reduced to $42.5 \pm 3.6 \%$ (fractions 5 and $6 ; P$ $=0.0007, n=6$ ), COP-associated $110 \mathrm{kDa}$ TrkA had increased to $37.3 \pm 3.2 \%$ (fractions 3 and $4 ; P=0.002$, $\mathrm{df}=$ 6), GN-associated $110 \mathrm{kDa}$ TrkA had increased to $20 \pm 1.7 \%$ (fractions 1 and $2 ; P<0.0001, \mathrm{df}=6$ ) and $140 \mathrm{kDa}$ TrkA was more clearly detected in GN membranes (fractions 1 and 2). Total TrkA (110kDa plus $140 \mathrm{kDa}$ ) was, therefore, reduced to $30.9 \pm 3.1 \%$ in ER membranes (fractions 5 and 6; $P=0.0001, \mathrm{df}=6$ ) and increased to $40.6 \pm 4 \%$ in GN membranes (fractions 1 and $2 ; P=0.0001, \mathrm{df}=6$ ). This change continued through 45 minutes and by 60 minutes resulted in a further reduction in ER-associated immature $110 \mathrm{kDa}$ TrkA to $28.8+2 \%$ (fractions 5 and 6 , versus 15 minutes $P<0.0001$, $\mathrm{df}=6$ ); $23.8 \pm 1.7 \%$ in COPmembranes (fractions 3 and 4 ) and a significant increase to $46.9 \pm 3.4 \%$ in GN membranes (fractions 1 and 2; verses 15 minutes $P<0.0001, \mathrm{df}=6$ ). This was associated with further increases, at both 45 and 60 minutes, in GNassociated (fractions 1 and 2) 140kDa TrkA levels. At 60 minutes, total TrkA (110kDa plus $140 \mathrm{kDa})$ levels had reduced to $13.4 \pm 1.2 \%$ in ER membranes (fractions 5 and 6; versus 15 minutes $P<0.0001, n=6$ ) and increased to $69 \pm 6.4 \%$ in GN membranes (Fractions 1 and 2; versus 15 minutes $P<0.0001, n=6$ ), indicating rapid $110 \mathrm{kDa}$ TrkA movement from ER to GN, in association with $140 \mathrm{kDa}$ TrkA maturation.

The trafficking of labelled TrkAIII differed considerably to that of immature $110 \mathrm{kDa}$ (Figure 4B). At 15 minutes, $61.5 \pm 5.2 \%$ of immature $100 \mathrm{kDa}$ TrkAIII was ER-associated (fractions 5 and 6), $28 \pm 2.4 \%$ COP-associated (fractions 3 and 4) and 10.4 $\pm 0.9 \%$ $\mathrm{GN}$-associated (fractions 1 and 2). By 30 minutes, ERassociated $100 \mathrm{kDa}$ TrkAIII had reduced to $26.9+2.3 \%$ (fractions 5 and $6 ; P<0.0001$, df $=6$ ), significantly increased to $53.5 \pm 4.6 \%$ in COP membranes (fractions 3 and $4 ; P<0.0001, \mathrm{df}=6$ ) and to $19.6 \pm 1.8 \%$ in GN membranes (fractions 1 and $2 ; P<0.0001$, df $=6$ ). At 45 minutes, $100 \mathrm{kDa}$ TrkAIII was $25.7 \pm 2.2 \%$ ER-associated (fractions 5 and 6; versus 15 minutes $P<0.0001$, df =6), 39.1 $\pm 3.3 \%$ COP-associated (fractions 3 and 4; significantly increased versus 15 minutes $P<0.0001, n$ $=6$; significantly reduced compared to 30 minutes $P<$ 0.0001 , $\mathrm{df}=6$ ), and $35.2 \pm 3.1 \% \mathrm{GN}$-associated (fractions 1 and 2; versus 30 minutes $P<0.0001, \mathrm{df}=6$ ). By 60 minutes $100 \mathrm{kDa}$ TrkAIII was $62 \pm 5.3 \%$ ER-associated (fractions 5 and 6; versus 45 minutes $P<0.0001$, df $=$ 6), 26 $\pm 2.2 \%$ COP-associated (fractions 3 and 4; versus 45 minutes $P<0.0001, \mathrm{df}=6)$ and only $12 \pm 1 \% \mathrm{GN}$ associated (fractions 1 and 2; versus 45 minutes; $P<$ 0.0001 , $\mathrm{df}=6$ ), indicating the retrograde transport of TrkAIII back to the ER.

CEP-701 (100nM: 3 hours pre-incubation prior to metabolic labelling, then throughout the time course) (Figure 4C), resulted in a pattern of $100 \mathrm{kDa}$ TrkAIII distribution that at 15 minutes was $69.7 \pm 6 \%$ ER-associated (fractions 5 and 6), $18 \pm 1.5 \%$ COP-associated (fractions 3 and 4 ) and $12.3 \pm 0.9 \% \mathrm{GN}$-associated (fractions 1 and 2 ), at 30 minutes was significantly reduced to $18.3 \pm 2.3 \%$ ER-associated (fractions 5 and 6; $P<0.0001, \mathrm{df}=6$ ) and significantly increased to $48.9 \pm 5.2 \%$ COP-associated (fractions 3 and $4 ; P<0.0001, \mathrm{df}=6$ ) and $32.5 \pm 3.5 \%$ GN-associated (fractions 1 and 2; $P<0.0001$, $\mathrm{df}=6$ ). Furthermore, a novel $120 \mathrm{kDa}$ TrkAIII species was detected in GN membrane fraction 1. Total TrkAIII $(100 \mathrm{kDa}$ plus $120 \mathrm{kDa})$ was, therefore, $15.8 \pm 1.6 \%$ ERassociated (fractions 5 and 6 ), $41.9 \pm 3.6 \%$ COP-associated (fractions 3 and 4 ) and $41.3 \pm 3.6 \% \mathrm{GN}$-associated (fractions 1 and 2). By 60 minutes, CEP-701 had caused a significant reduction in ER-associated TrkAIII to 
$30.9 \pm 3.1 \%$ (fractions 5 and 6 ; versus untreated controls; $p<0.0001, n=6$ ) and to $23.8 \pm 2.4 \%$ in COP membranes (fractions 3 and 4; versus 30 minutes $P<0.0001$, df $=$ $6)$, associated with a significant increase in $\mathrm{GN}$-associated
TrkAIII to $45.1 \pm 4.5 \%$ (fractions 1 and 2; versus 30 minutes, $P=0.0045$, df $=6$ ). This was associated with a marked increase in $120 \mathrm{kDa}$ TrkAIII expression, which was distributed $58.7 \pm 7 \%$ in COP-negative GN membrane

A
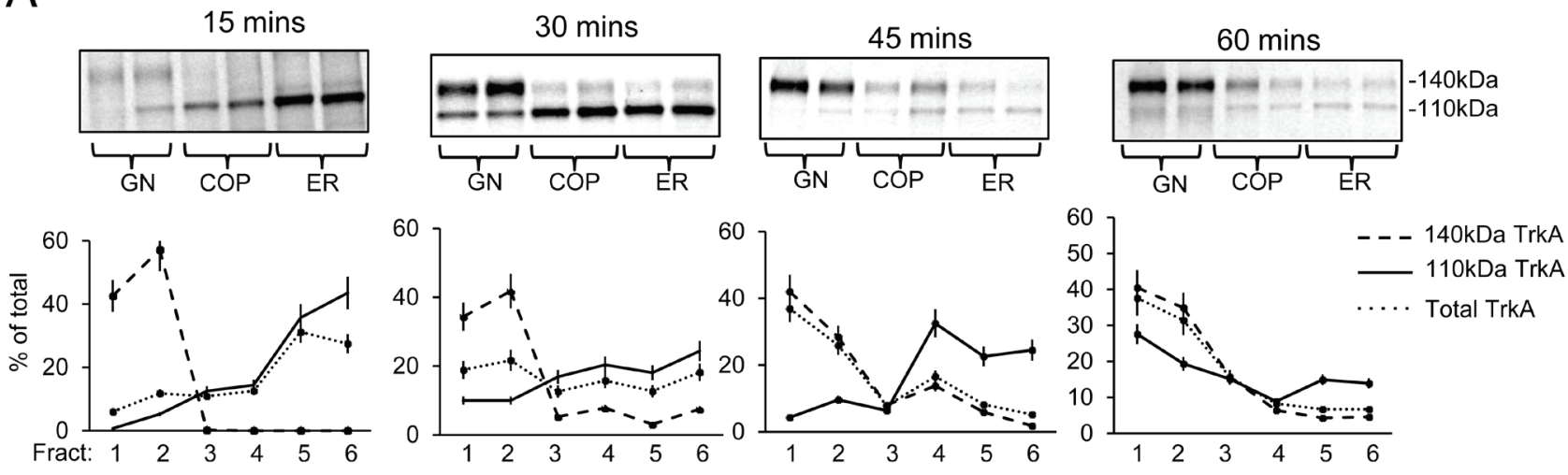

B

\section{TrkAlll SH-SY5Y}
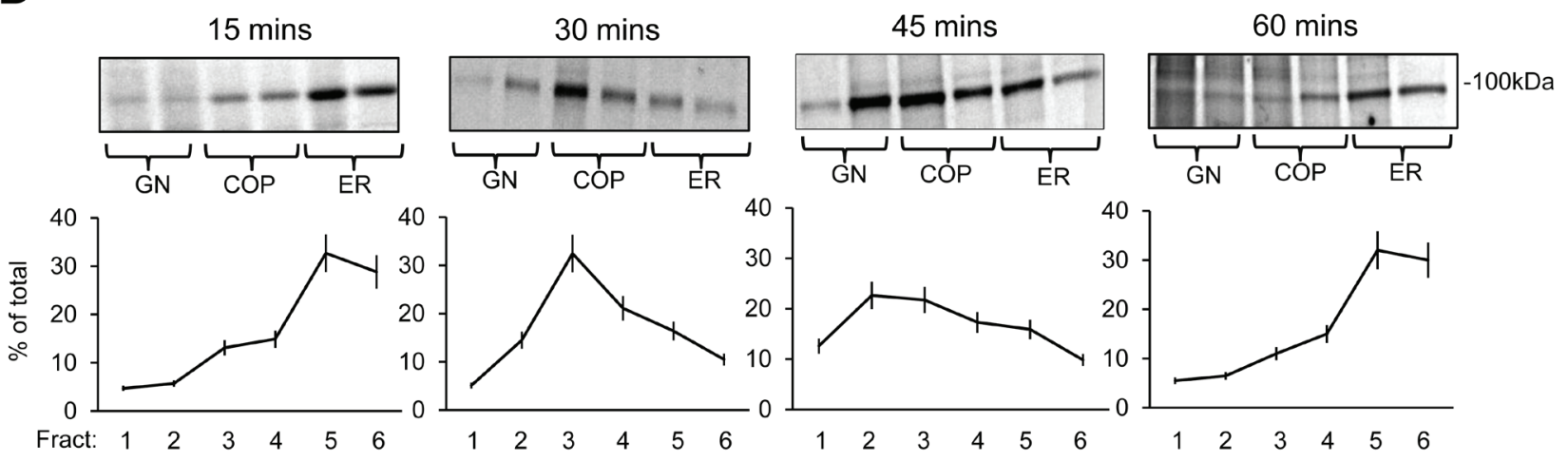

C

TrkAIII SH-SY5Y (CEP)
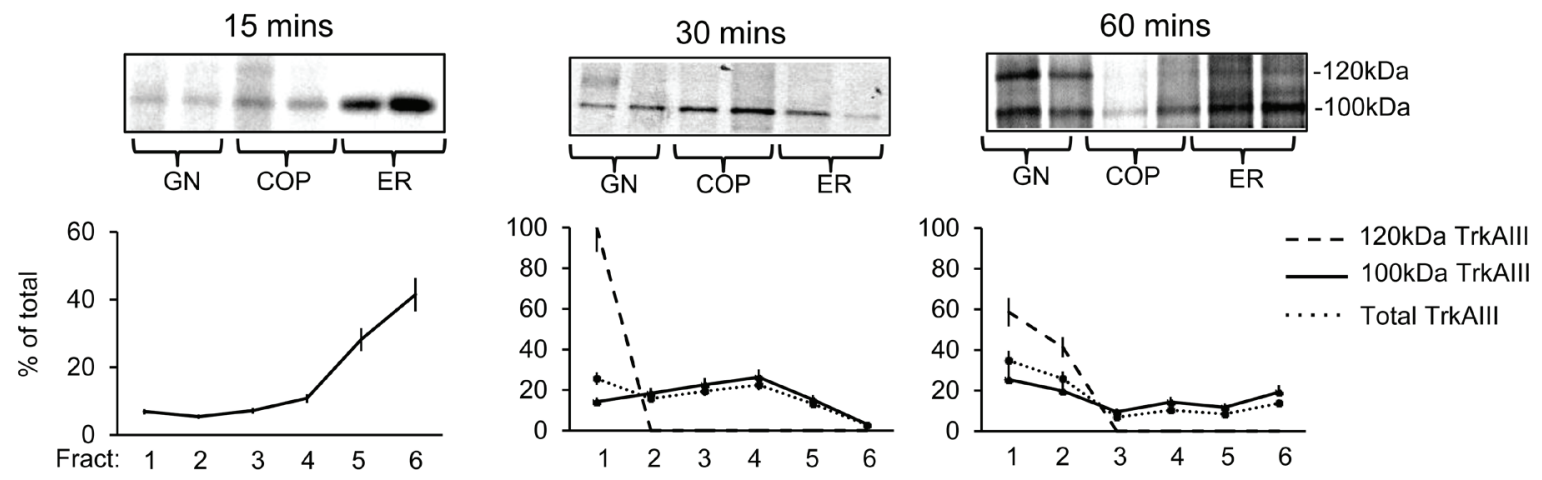

Figure 4: TrkAIII but not TrkA returns to the ER. Representative autoradiographs plus line graphs demonstrating time-dependent changes in the distribution of pulse labelled $110 \mathrm{kDa} / 140 \mathrm{kDa}$ TrkA and $100 \mathrm{kDa} / 120 \mathrm{kDa}$ TrkAIII in density-gradient ultra-centrifugation purified GN, COP vesicle and ER membrane fractions from: A. untreated TrkA SH-SY5Y cells; B. untreated TrkAIII SH-SY5Y cells; and C. TrkAIII SH-SY5Y cells treated with 100nM CEP-701 (CEP), at the times indicated in minutes, following metabolic pulse labelling (presented as the mean \pm s.d. percentage of total, derived from densitometric analysis of autoradiographs from independent experiments, performed in duplicate). 
fraction 1 and $41.3 \pm 4.9 \%$ in COP positive GN membrane fraction 2. Total TrkAIII levels (100kDa plus $120 \mathrm{kDa})$ were, therefore, significantly reduced to $22.1 \pm 2.9 \%$ in ER membranes (fractions 5 and 6; versus untreated controls at 60 minutes $P<0.0001, \mathrm{df}=6$ ) and to $17.1 \pm 2.2 \%$ in COP membranes (fractions 3 and 4; versus 60 minutes untreated controls; $P=0.0012, \mathrm{df}=6$ ), and significantly increased to $60.6 \pm 7.8 \%$ in GN membranes (fractions 1 and 2; versus 60 minutes untreated controls $P<0.0001$, df $=6$ ). This indicates that CEP-701 promotes TrkAIII trafficking from the ER to GN, resulting in GN-associated $120 \mathrm{kDa}$ TrkAIII maturation.

\section{Spontaneous TrkAIII activation occurs within the ERGIC/COPI vesicle compartment}

In TrkAIII SH-SY5Y cells, confocal IF confirmed a close relationship between Y490 phosphorylated TrkAIII and the ERGIC marker ERGIC-53, when compared to the cis-GN marker GM130, the trans-GN marker TGN46 and the ER marker calnexin (Figure 5A), confirming our previous report using non-confocal IF [3].

Brefeldin A (BFA; $5 \mu \mathrm{g} / \mathrm{ml}$ for 6 hours) abrogated TrkAIII tyrosine phosphorylation, assessed by both indirect IF (Figure 5B) and Western blotting of whole cell extracts (Figure 5C). BFA caused re-distribution of GM130 in TrkAIII SH-SY5Y cells, consistent with disruption of a constitutively intact GN (Figure 5D).

In co-immunoprecipitation assays, tyrosine phosphorylated TrkAIII pulled down COPI from densitygradient ultracentrifugation purified membrane fraction 3 , whereas non-phosphorylated TrkA did not, confirming a different relationship between COP-I, non-phosphorylated TrkA in TrkA SH-SY5Y cells and tyrosine phosphorylated TrkAIII in TrkAIII SH-SY5Y cells, respectively (Figure 5E).

\section{TrkA inhibitors promote $120 \mathrm{kDa}$ TrkAIII maturation}

Different TrkA inhibitors were compared in their capacity to promote $120 \mathrm{kDa}$ TrkAIII maturation. The TrkA inhibitors CEP-701, K252a, Go6976 and GW441756 [17-21] all abrogated TrkAIII tyrosine phosphorylation and promoted $120 \mathrm{kDa}$ TrkAIII maturation (Figure 6A). In contrast, the hyper-activation of TrkAIII induced by sodium orthovanadate did not result in $120 \mathrm{kDa}$ TrkAIII maturation, supporting an inverse relationship between TrkAIII tyrosine phosphorylation and $120 \mathrm{kDa}$ maturation (Figure 6B). In IF studies, all TrkA inhibitors decreased TrkAIII staining intensity and promoted more diffuse staining without evidence of cell surface expression, whereas sodium orthovanadate did not alter TrkAIII staining intensity or distribution (Figure 6C). Combination of TrkA inhibitors with the Hsp90 inhibitor Geldanamycin
$(\mathrm{GA}, 1 \mu \mathrm{M})$ further reduced TrkAIII staining intensity but in the presence of the proteasome inhibitor MG$132(1 \mu \mathrm{M})$ TrkAIII staining intensity was restored in association with re-distribution to intercellular junctions and cellular projections, consistent with potential cell surface expression (Figure 6C). These data suggest that TrkA inhibitors and GA promote TrkAIII degradation at the proteasome.

\section{Nocodozol and GA inhibit 120kDa TrkAIII Maturation}

MG-132 (1 $\mu \mathrm{M}$ for 3 and 6 hours) induced low-level $120 \mathrm{kDa}$ TrkAIII expression, suggesting that in TrkAIII SH-SY5Y cells a proportion of TrkAIII must arrive at the GN but upon $120 \mathrm{kDa}$ maturation is degraded at the proteasome and, therefore, not readily detected (Figure 7A).

Nocodozol $(0.1 \mu \mathrm{M})$ abrogated $120 \mathrm{kDa}$ TrkAIII maturation induced by CEP-701 in TrkAIII SH-SY5Y cells and also abrogated 140kDa TrkA maturation in TrkA SH-SY5Y cells (Figure 7B), confirming that both $120 \mathrm{kDa}$ TrkAIII and $140 \mathrm{kDa}$ TrkA maturation is microtubuledependent, which is consistent with COPI vesicle transport to the GN $[22,23]$.

GA ( $1 \mu \mathrm{M}$ for 12 hours) inhibited TrkAIII tyrosine phosphorylation, did not reduce $100 \mathrm{kDa}$ TrkAIII levels nor induce $120 \mathrm{kDa}$ TrkAIII expression (Figure 7C) but did inhibit 120kDa TrkAIII maturation induced by CEP-701 (Figure 7D). MG-132 not only reversed this GA-inhibitory effect but also augmented $120 \mathrm{kDa}$ TrkAIII expression above that induced by CEP-701 alone (Figure 7D), indicating that GA enhances $120 \mathrm{kDa}$ TrkAIII degradation at the proteasome. We also confirm our previous report [2], that MG-132 reverses GA abrogation of $140 \mathrm{kDa}$ TrkA expression in TrkA SH-SY5Y cells (Figure 7D). GA did not reduce $100 \mathrm{kDa}$ TrkAIII or $110 \mathrm{kDa}$ TrkA levels, indicating that these immature receptor forms are relatively insensitive to degradation at the proteasome and are not stabilized by GA-sensitive interaction with Hsp90.

The thiol reducing agent dithiothreitol (DTT) inhibits protein folding, promotes protein retention within the ER and prevents protein trafficking to the GN [24]. DTT abrogated both CEP-701-induced 120kDa TrkAIII maturation and constitutive $140 \mathrm{kDa}$ TrkA maturation, without reducing immature receptor levels (Figure 7E, left panel). This effect was not inhibited by MG-132, confirming it to be proteasome-independent, consistent with the necessity for transport to the GN for both $120 \mathrm{kDa}$ TrkAIII and 140kDa TrkA maturation (Figure 7E)

\section{DISCUSSION}

In this study, we characterise the re-localisation mechanism responsible for spontaneous activation 

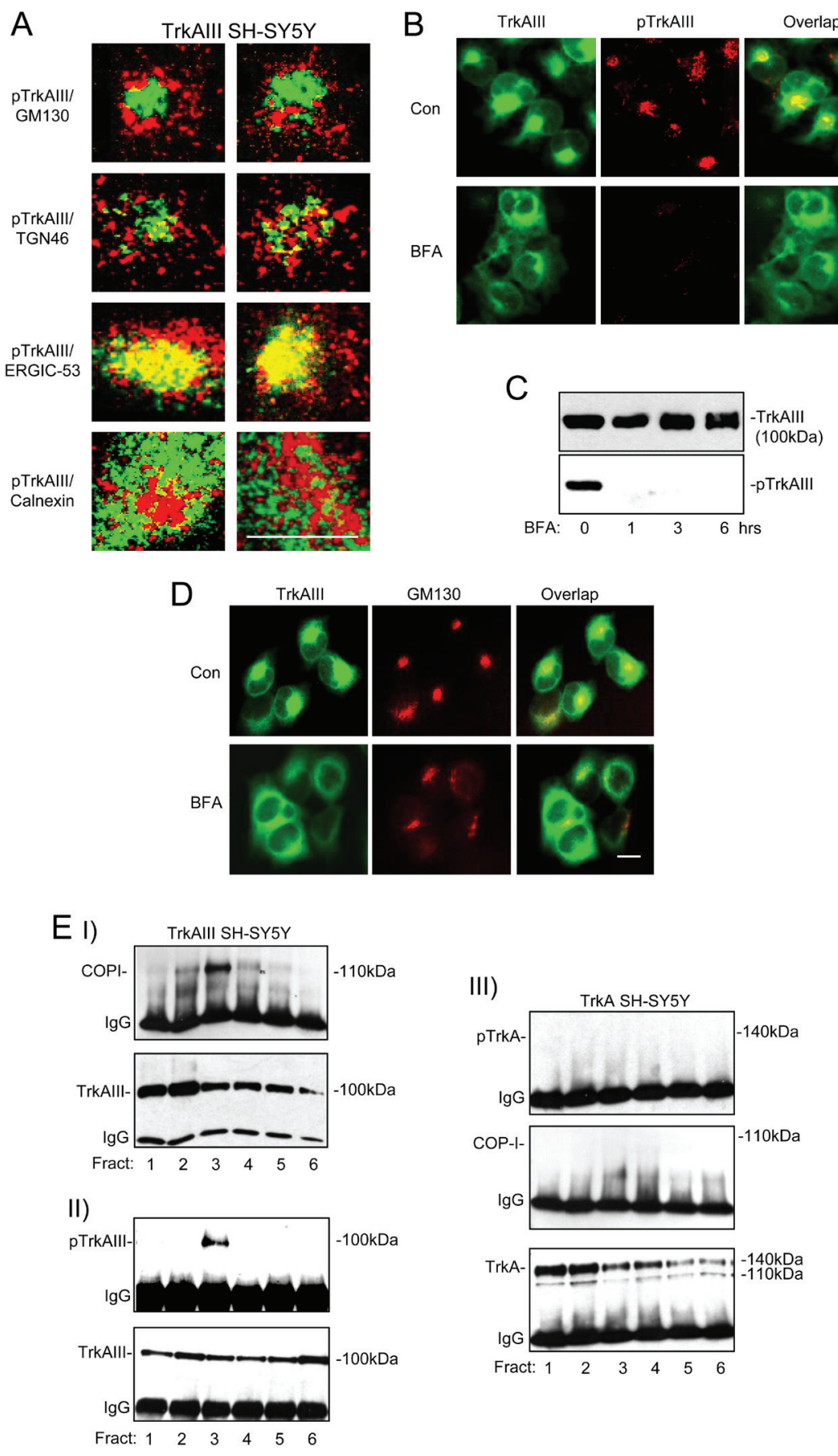

Figure 5: Spontaneous TrkAIII activation occurs within the ERGIC/COPI compartment. Representative confocal IF micrographs demonstrating different degrees of association (yellow) between Y490-phosphorylated TrkAIII (pTrkAIII) (red) and GM130 (green) (top panels); pTrkAIII (red) and TGN46 (green) (2 ${ }^{\text {nd }}$ panels); pTrkAIII (red) and ERGIC-53 (green) ( $3^{\text {rd }}$ panels); and pTrkAIII (red) and Calnexin (green) (bottom panels) in individual representative TrkAIII SH-SY5Y cells (magnification x100; bar $=10 \mu \mathrm{m})$. B. Indirect non-confocal IF, demonstrating the degree of overlap (yellow) between TrkAIII (green) and phosphorylated TrkAIII (red) in untreated TrkAIII SH-SY5Y cells (Con) (top 3 panels) and BFA-treated $(0.1 \mu \mathrm{M}$ for $3 \mathrm{hr}$ ) TrkAIII SH-SY5Y cells (bottom 3 panels; bar $=10 \mu \mathrm{m})$. C. Western blots of whole cell extracts $(30 \mu \mathrm{g})$, demonstrating the effect of BFA $(0.1 \mu \mathrm{M}$ for $0,1,3$ and 6 hours $)$ on total and tyrosine phosphorylated TrkAIII. D. Indirect non-confocal IF, demonstrating overlap (yellow) and general distribution of TrkAIII (green) and GM130 (red) in untreated (Con) and BFA-treated $(0.1 \mu \mathrm{M}$ for $3 \mathrm{hr})$ TrkAIII SH-SY5Y cells. Western blots demonstrating: EI. TrkAIII co-immunoprecipitation of COP-I in density gradient ultracentrifugation-purified TrkAIII SH-SY5Y membranes; EII. The presence of tyrosine phosphorylated TrkAIII in TrkAIII immunoprecipitates from the same TrkAIII SH-SY5Y membrane preparation and EIII. The absence of TrkA/COPI co-immunoprecipitation and TrkA tyrosine phosphorylated in density gradient ultracentrifugation-purified intracellular membranes from TrkA SH-SY5Y cells. 
A

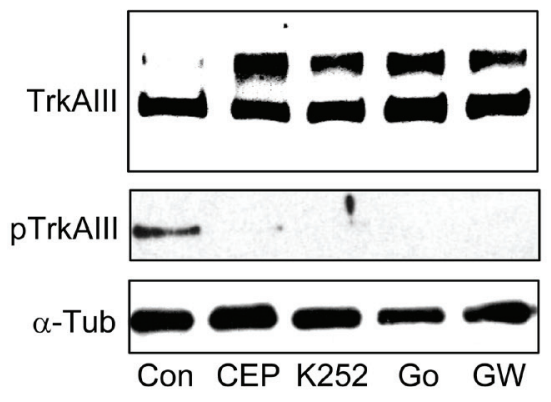

B

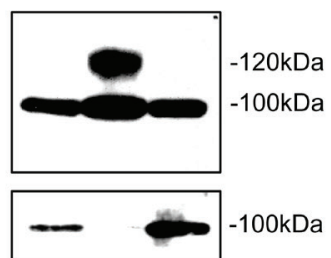

Con CEP Van

C

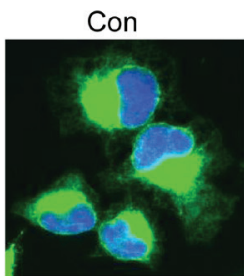

CEP

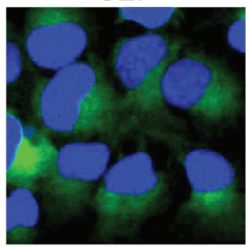

Go

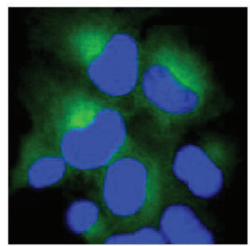

GW

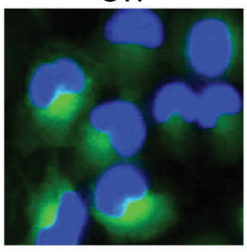

K252

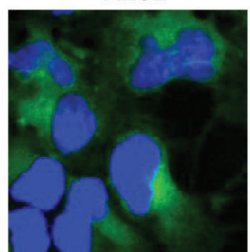

GA

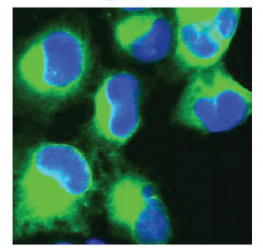

CEP/GA

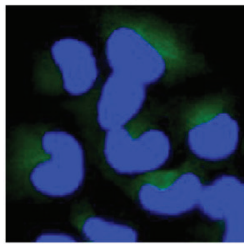

Go/GA

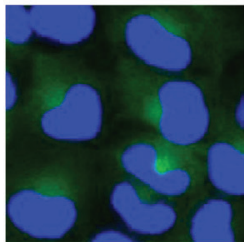

GW/GA

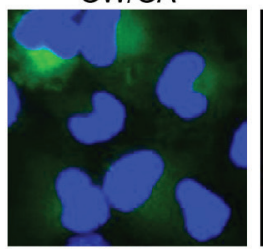

K252/GA

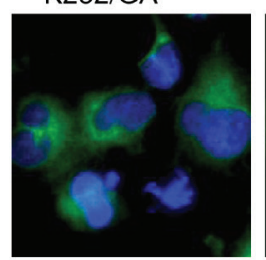

MG

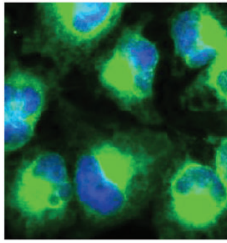

CEP/GA/MG

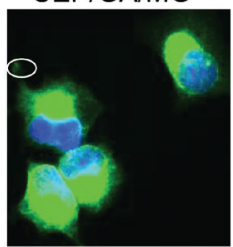

Go/GA/MG

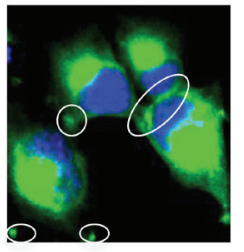

GW/GA/MG

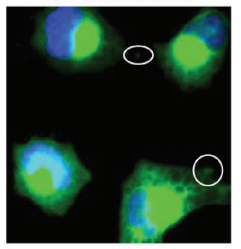

K252/GA/MG

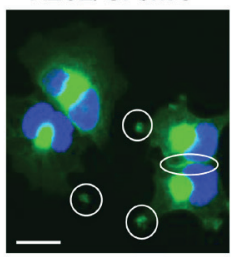

Figure 6: TrkA inhibitors promote 120kDa TrkAIII maturation and degradation. A. Western blots demonstrating the levels of $100 \mathrm{kDa}$ and $120 \mathrm{kDa}$ TrkAIII (TrkAIII), tyrosine phosphorylated TrkAIII (pTrkAIII) and $\alpha$-tubulin in total extracts $(30 \mu \mathrm{g})$ from untreated (con); CEP-701-treated (CEP; 100nM for 6 hours); K252a-treated (K252; 100nM for 6 hours); Go6076-treated (Go; 100nM for 6 hours) and GW441756-treated (GW; 100nM for 6 hours) TrkAIII SH-SY5Y cells. B. Western blots comparing the effects of CEP-701 (CEP) and sodium orthovanadate (Van) on $100 \mathrm{kDa}$ and $120 \mathrm{kDa}$ TrkAIII levels and TrkAIII tyrosine phosphorylation (pTrkAIII). C. Indirect IF demonstrating changes in TrkAIII IF intensity and distribution in TrkAIII SH-SY5Y cells treated with CEP-701 (CEP), Go6976 (Go), GW441756 (GW) and K252a (K252), alone or in combination with GA plus or minus MG-132 (MG). Potential sites of cell surface TrkAIII are circled $($ bar $=10 \mu \mathrm{m})$. 
A

TrkAIII SH-SY5Y

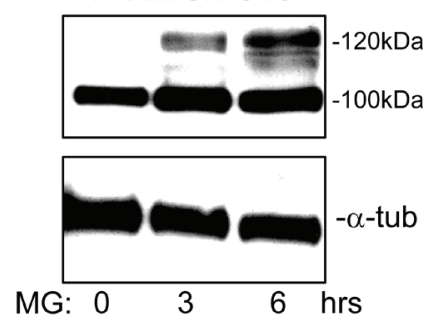

B

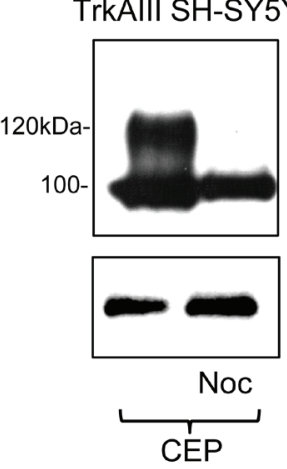

C
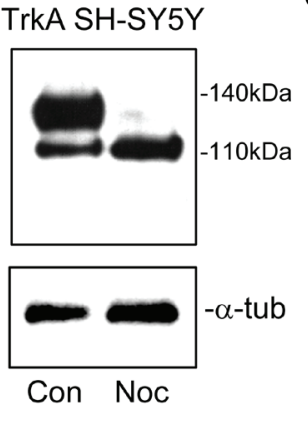

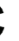
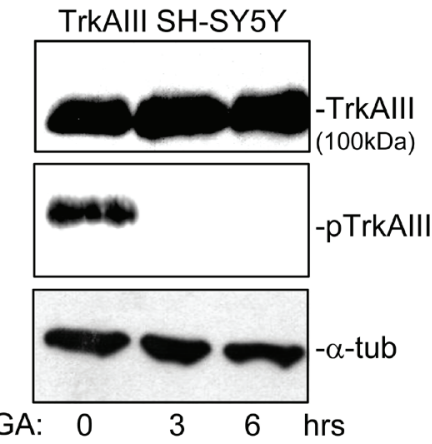

D

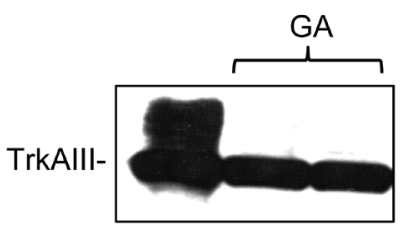

TrkAlll SH-SY5Y
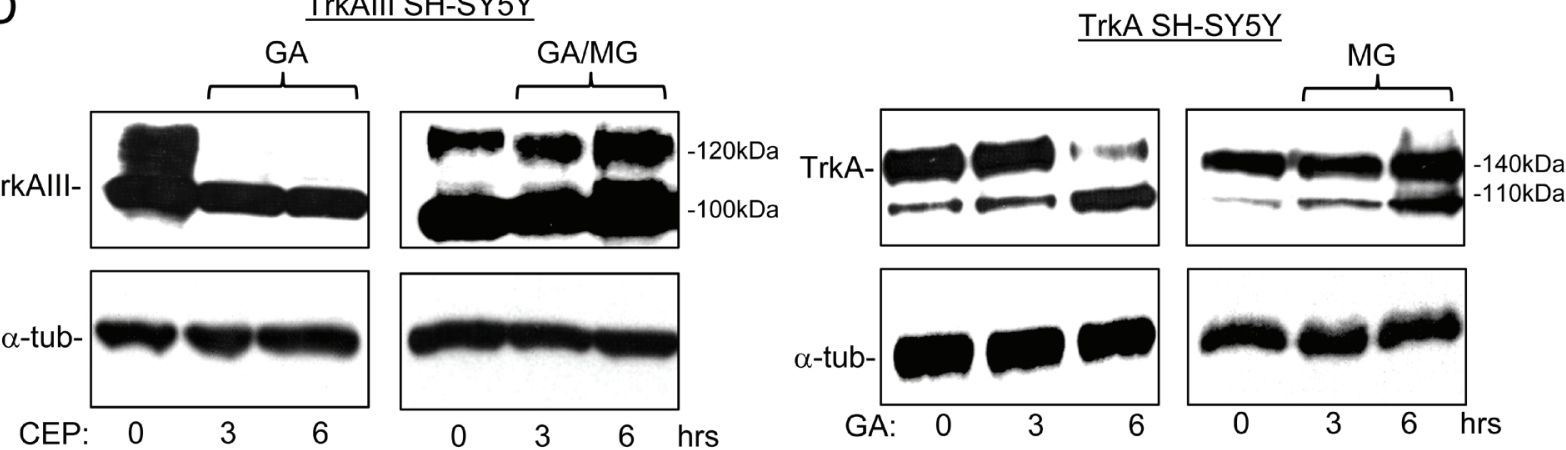

E

TrkAIII SH-SY5Y
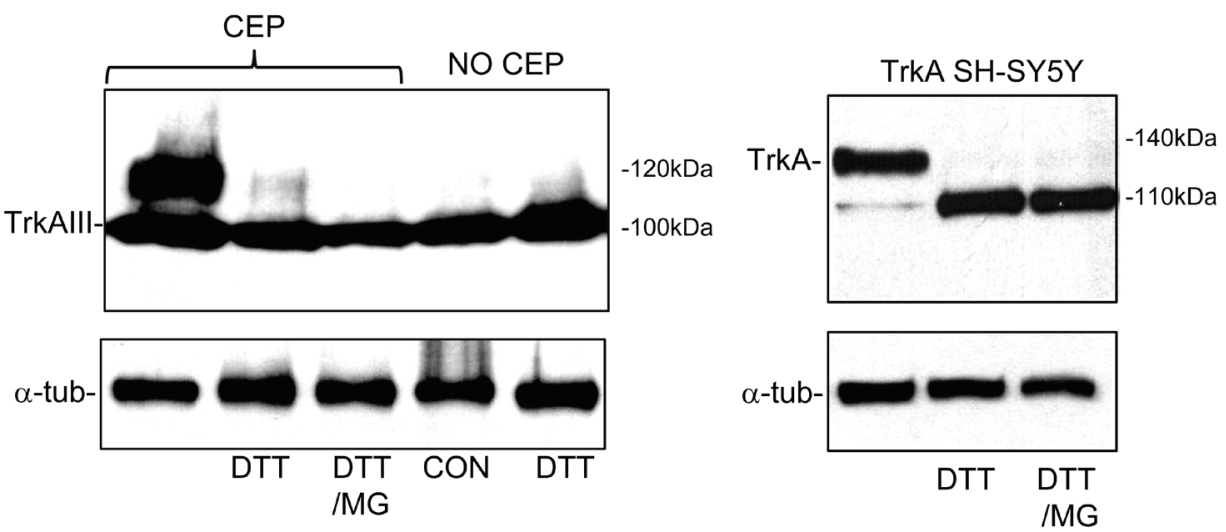

Figure 7: 120kDa TrkAIII is degraded at the proteasome, is microtubule-dependent and GA-sensitive. Western blots demonstrating: A. the effect of MG-132 (1 $\mu \mathrm{M}$ for 3 and 6 hours) on 100kDa TrkAIII, $120 \mathrm{kDa}$ TrkAIII and $\alpha$-tubulin ( $\alpha$-tub) levels in TrkAIII SH-SY5Y cells; B. the effect of Nocodozol (Noc; $100 \mu \mathrm{M}$ for 6 hours) upon 100kDa TrkAIII, 120kDa TrkAIII and $\alpha$-tubulin ( $\alpha$-tub) levels in CEP-701-treated (CEP) TrkAIII SH-SY5Y cells (left panel) and upon 110kDa TrkA, 140kDa TrkA and $\alpha$-tubulin levels in TrkA SH-SY5Y cells (right panel); C. The effect of Geldanamycin (GA; $1 \mu \mathrm{M}$ for 3 and 6 hours) on 100kDa TrkAIII, 120kDa TrkAIII, tyrosine phosphorylated TrkAIII (pTrkAIII) and $\alpha$-tubulin levels in TrkAIII SH-SY5Y cells; D. The effect of Geldanamycin (GA; $1 \mu \mathrm{M}$ for 3 and 6 hours) plus or minus MG-132 (MG; $1 \mu \mathrm{M}$ for 3 and 6 hours) on $100 \mathrm{kDa}$ TrkAIII, $120 \mathrm{kDa}$ TrkAIII and $\alpha$-tubulin ( $\alpha$-tub) levels in TrkAIII SH-SY5Y cells pre-treated with CEP-701 (CEP; 100nM for 6 hours) to induced 120kDa TrkAIII expression (Left 4 panels), plus the effect of the same combinations of GA plus or minus MG132, in non-CEP-701 treated TrkA SH-SY5Y cells (right 4 panels); E. The effect of DTT $(100 \mu \mathrm{M}$ for 6 hours) plus or minus MG-132 (MG; $1 \mu \mathrm{M})$ on 100kDa TrkAIII, $120 \mathrm{kDa}$ TrkAIII and $\alpha$-tubulin levels in CEP-701 pretreated (CEP; 100nM for 6 hours) and non-CEP-701 treated TrkAIII SH-SY5Y cells (Left panels) and in untreated TrkA SH-SY5Y cells (right panels). 
and subsequent oncogenic activity of the TrkAIII oncoprotein in SH-SY5Y NB cells. Exons 6-7 skipping that characterises TrkAIII, eliminates the D4 IG-like domain and several N-glycosylation sites involved in optimising intracellular trafficking and reducing spontaneous activation potential, increasing intracellular accumulation and facilitating spontaneous activation within the ERGIC/COPI vesicle compartment. This, in turn, promotes retrograde TrkAIII transport back to the ER and reduces anterograde transport to the GN, setting up a self-perpetuating mechanism for intracellular TrkAIII entrapment, accumulation, spontaneous activation and oncogenic Akt signalling from this alternative localisation. TrkA tyrosine kinase inhibitors abrogate this mechanism and promote TrkAIII transport to the GN, resulting in GNassociated TrkAIII maturation to a $120 \mathrm{kDa}$ species that is more readily degraded at the proteasome.

The marked difference in intracellular trafficking, cell surface expression and spontaneous activation exhibited by TrkA and TrkAIII in SH-SY5Y cells confirms the critical role played exons 6 and 7 encoded sequence in optimising intracellular trafficking from the ER to the GN, reducing intracellular spontaneous activation potential and promoting receptor maturation, an essential step in cell surface expression (this study and [2]). In the case of TrkA, this results in two predominant inactive steady state pools, one within the GN and the other at the cell surface (this study and [1-3]) both maintained inactive by a full complement of spontaneous activation inhibitory D4 and D5 IG-like domains, a full N-glycosylated status that reduces aberrant accumulation within the ERGIC, $\mathrm{N}$-glycan maturation that further reduces aberrant intracellular accumulation by promoting translocation to the cell surface $[1-4,7,8-10]$ and, in the case of cell surface TrkA, endogenous PTPase activity [2]. In contrast to TrkA, immature N-glycosylated $100 \mathrm{kDa}$ TrkAIII exits the ER but fails to adequately reach the GN. As a consequence, TrkAIII does not undergo significant GNassociated N-glycan maturation, fails to reach the cell surface and becomes entrapped between the ER and ERGIC intracellular membrane compartments, within which it accumulates and undergoes spontaneous ERGIC/ COPI-associated activation.

Metabolic labelling comparisons indicated that whilst TrkA and TrkAIII both exit the ER, TrkAIII recycles back to the ER from the ERGIC, resulting in entrapment and accumulation, whereas TrkA moves from the ERGIC to the GN. TrkAIII recycling from the ERGIC to the ER was prevented by TrkA inhibitors, which promoted TrkAIII trafficking to the GN, resulting in GNassociated TrkAIII N-glycan maturation. This indicates that retrograde recycling of TrkAIII back to the ER and reduced transport to the GN is TrkAIII activity-dependent. Subsequent TrkAIII entrapment and accumulation within the pre-GN compartment, combined with omission of the D4 IGC1 spontaneous activation prevention domain, further augment the potential for spontaneous intracellular activation. This novel activation-regulated re-localisation mechanism differs to that utilised by mutated FLT and Kit RTK oncogenes, which fail to satisfy ER quality control and are retained and activated within the ER [10-12], and the Trk-T3 oncogene that is retained and activated within the ERES/COPII compartment as a result of chimeric TFG sequences involved in COPII vesicle formation [13].

TrkAIII activation within an ERGIC/COPI vesicle context was confirmed using BFA, an inhibitor of ARF-1dependent COPI vesicle formation [21], which abrogated ERGIC-associated TrkAIII activation, was supported by the close relationship exhibited between COP-I positive membranes and tyrosine phosphorylated TrkAIII and by co-immunoprecipitation of tyrosine phosphorylated TrkAIII and COP-I from the same membrane fraction. The fact that BFA inhibited TrkAIII activity but did not promote TrkAIII $120 \mathrm{kDa}$ maturation is explained by its capacity to disrupt the GN (this study and [21]), which would prevent the transport of inactivated TrkAIII to the GN. BFA does not disrupt the ERGIC [25], further supporting the hypothesis of ERGIC-associated TrkAIII activation within a COP-I vesicle context but does prevent COP-II vesicle formation [21]. We do not exclude, therefore, a potential role for COP-II vesicles in regulating the spontaneous activation of TrkAIII. BFA inhibition of spontaneous TrkAIII activation in SH-SY5Y cells differs to a report that ERGIC-associated spontaneous TrkA activation in human kidney 293 cells is insensitivity to BFA and, therefore, COPI-independent [14].

The mechanism through which activated TrkAIII recycles back to the ER remains to be elucidated. TrkAIII does not contain KDEL-like sequences, suggesting a KDEL-R-independent mechanism but could complex with KDEL-cargos in active-form to facilitate retrograde transport in KDEL-R/COPI vesicles [26, 27]. KDEL-R is a G-protein coupled receptor (GPCR) and induces Arf1dependent COPI vesicle formation upon cargo binding [27]. GPRCs activate immature intracellular TrkA [28-30], suggesting an intriguing possibility that cargo-activated KDEL-Rs may activate TrkAIII, resulting in retrograde transport of KDEL-R/COPI vesicles containing KDELRactivated TrkAIII. Although immature TrkA receptors were not activated under similar conditions, this may explain the selective activation of TrkAIII within the ERGIC/COPI compartment. We are investigating this possibility.

Alternatively, the recycling of activated TrkAIII from the ERGIC to ER may result from motor proteindriven, MT minus-end, retrograde receptor transport, analogous to that described for the retrograde transport of ligand-activated cell surface TrkA receptors [31-33]. Such a mechanism would not only explain recycling back to the ER but also the accumulation of active TrkAIII at the centrosome [3]. Whatever the mechanism, the net result is self-perpetuating TrkAIII re-localisation to the ER/ 
ERGIC compartment, with the subsequent combination of entrapment and accumulation of this compromised receptor augmenting the potential for spontaneous activation.

TrkAIII activation within ERGIC/COPI compartment differs to activation of the TrkT3 oncogene, which occurs within the ERES/COPII compartment [13]. This can be explained by different re-localisation mechanisms. TrkT3 is trapped within the ERES/COPII compartment by chimeric TFG sequences, not present in TrkAIII, which regulate COPII vesicle formation [13]. Both mechanism, however, result in spontaneous activation and oncogenic Akt signalling from their respective locations, confirming that TrkA oncoproteins, compromised either by mutation or alternative splicing, may re-localise to different intracellular membranes but become activated when they accumulate above the local threshold for spontaneous activation.

In contrast to TrkAIII, TrkA did not exhibit spontaneous activation in SH-SY5Y cells, clearly implicating the extracellular D4 IG-like domain and associated N-glycosylation sites omitted from TrkAIII, in preventing spontaneous intracellular TrkA activation. This most likely depends upon the optimisation of intracellular TrkA trafficking to the GN, GN-associated $\mathrm{N}$-glycan maturation and subsequent expression at the cell surface [7-9]. The optimisation of intracellular TrkA trafficking to the GN appears to be key in this respect, as illustrated by the recent report of spontaneous ERGICassociated activation of immature TrkA in human kidney 293 cells [14]. This cell specific effect, characterised by aberrant TrkA accumulation within the ERGIC above the spontaneous activation threshold, results in GN disruption, which promotes the entrapment of immature TrkA within the ERGIC, illustrating the absolute requirement for optimised intracellular trafficking to the GN in preventing spontaneous intracellular activation [14].

TrkAIII did not exhibit spontaneous activation throughout the ER but could be activated throughout the ER by the PTPase inhibitor sodium orthovanadate, implicating ER PTPases in maintaining ER-associated TrkAIII inactive [2]. This indicates that active TrkAIII recycling back to the ER would be inactivated within the ER prior to being re-transport back to the ERGIC in COPII vesicles, the formation of which would be enhanced by TrkAIII-induced Akt signalling [1, 26]. Spontaneous TrkAIII activation within the GN was also minimal. This may reflect either the low level of TrkAIII in COPnegative GN membranes and/or the RTK inhibitory nature of the GN, which is exemplified by the steady state GNassociated pool of inactive TrkA, consistent with the GN function as natural reservoir for receptor tyrosine kinases during their maturation [34].

TrkA inhibitors promoted TrkAIII transport from the ER to GN, resulting in GN-associated TrkAIII maturation to $120 \mathrm{kDa}$, analogous to GN-associated TrkA maturation from $110 \mathrm{kDa}$ to $140 \mathrm{kDa}$ (this study and $[1,3]$ ). In contrast, hyper-phosphorylation of TrkAIII induced by sodium orthovanadate did not result in $120 \mathrm{kDa}$ TrkAIII maturation, supporting an inverse relationship between TrkAIII activation and maturation. This bears similarity to ERGIC-associated activation of immature $110 \mathrm{kDa}$ TrkA receptors in human kidney 293 cells, which impedes $140 \mathrm{kDa}$ TrkA maturation by disrupting the GN [14]. Here, we found no evidence for GN disruption in TrkAIII SH-SY5Y cells, suggesting that impaired maturation of TrkAIII involves the reduced transport of activated TrkAIII to the GN, rather than GN disruption.

The proteasome inhibitor MG132 promoted lowlevel expression of $120 \mathrm{kDa}$ TrkAIII without influencing $100 \mathrm{kDa}$ TrkAIII levels in TrkAIII SH-SY5Y cells. This indicates that a small proportion of TrkAIII must constitutively arrive at the GN and mature to $120 \mathrm{kDa}$ but is not detected in the absence of MG132 due to degradation at the proteasome. Furthermore, MG132 augmented $120 \mathrm{kDa}$ TrkAIII levels induced by CEP-701, restored 120kDa TrkAIII expression to CEP-701-treated TrkAIII SH-SY5Y cells in which $120 \mathrm{kDa}$ TrkAIII expression had been abrogated by the Hsp90 inhibitor GA and, in the presence of TrkA inhibitors, caused the re-distribution of TrkAIII to cellular projections and intracellular junctions, suggesting cell surface expression. Together, these data indicate that: TrkA inhibitors promote GN-associated TrkAIII $120 \mathrm{kDa}$ maturation; $120 \mathrm{kDa}$ TrkAIII is more sensitive than $100 \mathrm{kda}$ TrkAIII to degradation at the proteasome; $120 \mathrm{kda}$ TrkAIII is stabilised by GA sensitive interaction the Hsp90 adding to our report that TrkAIII exhibits GA-sensitive interaction with Hsp90 [2], and that cell surface expression of $120 \mathrm{kDa}$ TrkAIII is prevented by its degradation at the proteasome.

In conclusion, we propose that the novel relocalisation mechanism characterised by spontaneous ERGIC/COPI-associated activation and subsequent retrograde recycling back to the ER is responsible for the entrapment, accumulation, spontaneous activation and subsequent oncogenic activity of TrkAIII and is initiated from the alternative location of the ERGIC/ COPI compartment. This highlights the importance of intracellular TrkAIII re-localisation for oncogenic activity, identifies the ERGIC/COPI compartment as a preferential site for spontaneous TrkAIII activation and provides an explanation for how TrkAIII, compromised by the omission of the D4 IGC1 spontaneous activationprevention domain, is able to overcome the threshold for spontaneous activation. The subsequent and altered oncogenic signalling through PI3K/Akt but not Ras/ MAPK results in increased survival, a pattern of malignant gene expression, increased genetic instability and promotion of stem cell-like behaviour rather than differentiation $[1,3,4,6,35]$, which together not only promote tumour progression but may also be involved in tumour initiation. TrkA tyrosine kinase inhibitors abrogate 
this mechanism by promoting the anterograde transport of inactivated TrkAIII to the GN, resulting in GN-associated TrkAIII maturation to a $120 \mathrm{kDa}$ form that is degraded at the proteasome, reducing both intracellular accumulation and oncogenic activity of the TrkAIII oncoprotein.

\section{MATERIALS AND METHODS}

\section{Cell lines and reagents}

PcDNA SH-SY5Y, TrkA SH-SY5Y, and TrkAIII SH-SY5Y cell lines were grown in recommended medium (RPMI or Dulbecco's modified Eagle's medium) supplemented with $10 \%$ foetal calf serum and appropriate antibiotics (phleomycin [Zeocin], penicillin, and streptomycin), as described previously [1-4]. Cell culture reagents, MG-132, geldanamycin, cytochalasin D, nocodozol, proteinase inhibitor Cocktail, Bis-benzimide tri-hydrochloride, propidium iodide, Histodenz ${ }^{\mathrm{TM}}$, NGF, K252a, Go6976 and GW441756 were purchased from Sigma-Aldrich (St. Louis MO). Endoglycosidase H was from Roche Italia (Milan, It). The pan Trk inhibitor CEP-701 was from Cephalon Inc. (West Chester, PA). Polyclonal anti-TrkA (C14) and monoclonal anti-Phosphotyrosine (pY99) antibodies were from SantaCruz (Santa Cruz, Ca). The polyclonal anti- $\alpha$-tubulin antibody was from Sigma (St. Louis, MO). Polyclonal antiphospho-Y674/675 TrkA, anti-COPI, COPII and mouse monoclonal anti-Calnexin and anti-TGN46 antibodies were from Abcam (Cambridge, UK). The polyclonal antiphospho-Y490 TrkA antibody was from Cell signalling (Danvers, MA). Mouse monoclonal anti-GM130 antibody was from BD Bioscience (San Jose, Ca). FITC and TRICconjugated secondary anti mouse and anti-rabbit IgG antibodies were from Jackson Immune Research (Bar Harbor, Maine). VectorMount ${ }^{\mathrm{TM}}$ medium was from Vector Laboratories (Berlingame, $\mathrm{Ca}$ ).

\section{Histodenz ${ }^{\mathrm{TM}}$ ultracentrifugation fractionation of internal cell membranes}

Briefly, cells were incubated with cytochalasin $\mathrm{D}(1 \mu \mathrm{g} / \mathrm{ml})$ and nocodozol $(0.1 \mu \mathrm{M})$ for 1 hour at $37^{\circ} \mathrm{C}$ prior to harvest, scraped into cold $\mathrm{PBS}$, washed in homogenisation buffer $\left(130 \mathrm{mM} \mathrm{KCl}, 5 \mathrm{mM} \mathrm{MgCl}_{2}\right.$, $25 \mathrm{mM}$ Tris- $\mathrm{HCl} \mathrm{pH} \mathrm{7.3)} \mathrm{and} \mathrm{homogenised} \mathrm{in} 500 \mu \mathrm{l}$ homogenisation buffer containing proteinase inhibitor cocktail (1mM 4-(-2 amino ethyl) benzene-sulfanylfluoride hydrochloride, $20 \mu \mathrm{M}$ leupeptin, $15 \mu \mathrm{M}$ pepstatin and $15 \mu \mathrm{M}$ chymostatin). Homogenates were centrifuged for 5 minutes at $1000 \mathrm{xg}$ at $4^{\circ} \mathrm{C}$, supernatants were collected and re-centrifuged at 1000xg for 5 minutes at $4^{\circ} \mathrm{C}$ to give the post nuclear supernatant (PNS). The PNS was adjusted to $500 \mu \mathrm{l}$ in homogenisation buffer and layered on top of a step-gradient from bottom to top, of $500 \mu \mathrm{l}$ of $40 \%, 1 \mathrm{ml}$ of $20 \% ; 1 \mathrm{ml}$ of $17.5 \%$ and $1 \mathrm{ml}$ of $15 \%$ Histodenz $^{\mathrm{TM}}$ (Sigma-Aldrich) in homogenisation buffer. Gradients were ultra centrifuged at $4^{\circ} \mathrm{C}$ in a Sw55Ti rotor for 60 minutes at 100,000xg and $500 \mu \mathrm{l}$ fractions collected (top to bottom) and mixed with $125 \mu \mathrm{l}$ of $5 \mathrm{x}$ detergent buffer (5\%NP-40, 2.5\% deoxycholate, $0.5 \%$ SDS, $250 \mathrm{mM}$ Tris- $\mathrm{HCl} \mathrm{pH} 8.0$ ). Fractions were analysed directly by Western blot or subjected to immunoprecipitation/ Western blot. Immunoprecipitates were washed with RIPA buffer (1\% NP-40, 0.5\% deoxycholate, $0.1 \%$ SDS, $150 \mathrm{mM} \mathrm{NaCl}$ and 50mM Tris- $\mathrm{HCl} \mathrm{pH} 8.0$ ); twice with high salt buffer (0.2\% SDS, $1 \%$ TritonX-100, 5mM EDTA, 500mM NaCl and $50 \mathrm{mM}$ Tris- $\mathrm{HCl} \mathrm{pH} 8.0)$ and once with TEN (50mM Tris- $\mathrm{HCl} \mathrm{pH} 8.0 ; 5 \mathrm{mM}$ EDTA and $150 \mathrm{mM} \mathrm{NaCl}$ ), prior to being subjected to reducing SDS PAGE/Western blotting.

\section{Immunoprecipitation and western blots}

Cells were extracted in lysis buffer (PBS containing $0.5 \%$ sodium deoxycholate, $1 \%$ NP40, $0.1 \%$ SDS, $1 \mathrm{mM}$ sodium orthovanadate, $1 \mathrm{mM}$ PMSF, $1 \mu \mathrm{g} / \mathrm{ml}$ of pepstatin A and Aprotinin), protein concentrations were calculated by Bradford Assay (Sigma-Aldrich) and extracts pre-cleared with $1 \mu \mathrm{g}$ pre-immune $\operatorname{IgG}\left(1\right.$ hour at $4^{\circ} \mathrm{C}$ ) and $20 \mu \mathrm{l}$ protein A Sepharose (Fast Flow, Sigma) for 20 minutes at $4{ }^{\circ} \mathrm{C}$. For immunoprecipitation, pre-cleared extracts (200-500 $\mu \mathrm{g}$ for stable transfectants) were incubated with primary antibody at a concentration of $0.1-1 \mu \mathrm{g} / 500 \mu \mathrm{g}$ total protein for 2-16 hours at $4^{\circ} \mathrm{C}$. Following incubation, $20 \mu \mathrm{l}$ Protein A Sepharose (Fast flow, Sigma-Aldrich) in lysis buffer, was added and reactions incubated for 60 minutes at $4{ }^{\circ} \mathrm{C}$. Protein sepharose/IgG conjugates were collected by centrifugation $(10,000 \mathrm{xg}$ for 5 minutes), washed 3 times in lysis buffer, re-suspended in SDS-PAGE sample buffer and subjected to reducing SDS-PAGE/Western blotting. Proteins were trans-blotted by electrophoresis onto Hybond $\mathrm{C}+$ nitrocellulose membranes (Amersham Int. UK) and membranes air-dried. Non-specific protein binding site were blocked by 2 hours incubation in 5\% non-fat milk in TBS, prior to incubation for 2-16 hours with primary antibodies diluted in blocking solution at $4^{\circ} \mathrm{C}$. Following incubation, membranes were washed in TBS, incubated for 20 minutes with secondary HRPconjugated antibody diluted in blocking solution and immunoreactive species detected by chemiluminescence reaction, as directed (Amersham Int., Bedford, UK).

\section{Metabolic labelling of TrkAI and TrkAIII}

Briefly, 3-4 million cells per time point, preincubated for 8 hours in the presence or absence of $100 \mathrm{nM}$ CEP-701, were incubated for 2 hour in methionine and cysteine-free medium prior to exposure for 30 minutes to $100 \mu \mathrm{Ci} / \mathrm{ml} \mathrm{S}^{35}$-labelled methionine/cysteine (Perkin- 
Elmer). Cells were then washed with pre-warmed complete medium and chased in excess $(1 \mathrm{mM})$ cold methionine/cysteine (Sigma-Aldrich), for different times. Cell aliquots were taken at each time point and subjected to Histoden $\mathrm{z}^{\mathrm{TM}}$ ultracentrifugation fractionation as described above, membrane fractions separated by regular reducing SDS-PAGE and dried gels were subjected to autoradiography. Densitometric analysis was performed using Image $\mathrm{J}$ software.

\section{Regular and confocal immunofluorescence (IF)}

Cells grown on Nunc glass chamber slides (SigmaAldrich) were washed in PBS, fixed in 37\% formaldehyde, permeabilized in cold methanol and processed for indirect immunofluorescence (IF). Slides were incubated for 1 $\mathrm{h}$ in blocking solution ( $1 \%$ bovine serum albumin in PBS- $0.03 \%$ TX100) and then for 2 to $16 \mathrm{~h}$ with primary antibody in blocking solution at room temperature. Slides were washed three times in PBS-0.03\% TX100, then incubated with secondary fluorochrome-conjugated antibody diluted in blocking solution for $1 \mathrm{~h}$ at room temperature, and then washed in PBS-0.03\% TX100 and mounted using Vector Mount. For confocal analysis, slides were examined under a Nikon Eclipse 80i confocal microscope and images elaborated using EZ-C1 Gold software (version 3.80). For non-confocal analysis, slides were examined under a Zeiss Axioplan 2 fluorescence microscope, with digital camera and Leica M500 Image Manager software.

\section{Statistical analysis}

Data were analysed statistically by Student's t-test. Statistical significance was associated with probabilities of $<0.05$.

\section{ACKNOWLEDGMENTS}

Foundation.

This work was supported by the Maugeri

\section{CONFLICTS OF INTEREST}

The authors declare no conflicts of interest.

\section{REFERENCES}

1. Tacconelli A, Farina AR, Cappabianca L, De Santis G, Tessitore A, Vetuschi A, Sferra R, Rucci N, Argenti B, Screpanti I, Gulino A, and Mackay AR. TrkA alternative splicing: a regulated tumor-promoting switch in human neuroblastoma. Cancer Cell. 2004; 6:347-360.

2. Farina AR, Tacconelli A, Cappabianca L. Cea G, Chioda
A, Romanelli A, Pensato S, Pedone C, Gulino A, and Mackay AR. The neuroblastoma tumour-suppressor TrkAI and its oncogenic alternative TrkAIII splice variant exhibit geldanamycin-sensitive interactions with Hsp90 in human neuroblastoma cells. Oncogene. 2009; 28:4075-4094.

3. Farina AR, Tacconelli A, Cappabianca L, Cea G, Panella S, Chioda A, Romanelli A, Pedone C, Gulino A, Mackay AR. The TrkAIII splice variant targets the centrosome and promotes genetic instability. Mol Cell Biol. 2009; 29:48124830.

4. Farina AR, Di Ianni N, Cappabianca L, Ruggeri P, Ragone M, Ianni G, Gulino A, and Mackay AR. TrkAIII promotes microtubule nucleation and assembly at the centrosome in SH-SY5Y neuroblastoma cells, contributing to an undifferentiated anaplastic phenotype. Biomed Res Int. 2013; http://dx.doi.org/10.1155/2013/740187.

5. Schramm A, Schowe B, Fielitz K, Heilmann M, Martin M, Marschall T, Koster J, Vandesompele J, Vermeulen J, de Preter K, Koster J, Versteeg R, Noguera R, Speleman F, Rahmann S, Eggert A, Morik K, and Schulte JH. Exon-level expression analyses identify MYCN and NTRK1 as major determinants of alternative exon usage and robustly predict neuroblastoma outcome. Br J Cancer 2012; 107:1409-1417.

6. Simpson AM, Iyer R, Mangino JL, Jennifer L. Mangino, Jane E. Minturn, Huaqing Zhao, Venkatadri Kolla, and Garrett M. Brodeur. TrkA3 isoform expression upregulates stem cell markers and correlates with worse outcome in neuroblastomas (NBs). Proc Adv Neuroblast Res 2012; p164 (POT055).

7. Watson FL, Porcionatto MA, Bhattacharyya A, Stiles $\mathrm{CD}$, and Segal RA. TrkA glycosylation regulates receptor localisation and activity. J Neurobiol. 1999; 39:323-336.

8. Collier E, Carpentier JL, Beitz L, Caro H, Taylor S I, and Gordon P. Specific glycosylation site mutations of insulin receptor alpha subunit impairs intracellular transport. Biochemistry. 1993; 32:7818-7823.

9. Arevalo JC, Conde B, Hempstead BL, Chao MV, MartinZanca D, and Perez P. TrkA immunoglobulin-like ligand binding domains inhibit spontaneous activation of the receptor. Mol Cell Biol. 2000; 20:5908-5916.

10. Choudhary C, Olsen JV, Brandts C, Cox J, Reddy PNG, Bohmer FD, Gerke V, Schmidt-Arras D-E, Berdel WE, Muller-Tidow C, Mann M, and Serve H. Mislocalized activation of oncogenic RTKs switches downstream signaling outcomes. Mol Cell. 2009; 36:326-339.

11. Schmidt-Arras D, Bohmer S-A, Koch S, Muller JP, Blei L, Cornils H, Bauer R, Korasikha S, Thiede C, and Bohmer F-D. Anchoring of FLT3 in the endoplasmic reticulum alters signaling quality. Blood. 2009; 113:3568-3576.

12. Corbacioglu S, Killic M, Westhoff M-A, Reinhardt D, Fulda S, and Debatin K-M. Newly identified c-KIT receptor tyrosine kinase ITD in childhood AML induces ligandindependent growth and is responsive to a synergistic effect of imatib and rapamycin. Blood. 2006; 108:3504-3513. 
13. Johnson A, Bhattacharya N, Hanna M, Pennington JG, Schuh A, Wang L, Otegui MS, Stagg SM, and Audhya A. TFG clusters COPII-coated transport carriers and promotes early secretory pathway organization. EMBO J. 2015; 34:811-827.

14. Schecterson LC, Hudson MP, Ko M, Philippidou P, Akmentin W, Wiley J, Rosenblum E, Chao MV, Halegoua $\mathrm{S}$, Bothwell M. Trk activation in the secretory pathway promotes Golgi fragmentation. Mol Cell Neurosci. 2010; 43:403-413.

15. Elbein AD. Inhibitors of the biosynthesis and processing of N-linked oligosaccharides. CRC Crit Rev Biochem. 1984; 16:21-49.

16. Tarentino AL, Plummer TH, and Jr. Maley F. The release of intact oligosaccharides from specific glycoproteins by endo$\beta$-N-acetylglucosaminidase H. J Biol Chem. 1974; 249:818824.

17. Al-Obeidi FA, and Lam KI. Development of inhibitors for protein tyrosine kinases. Oncogene. 2000; 19:5690-5701.

18. Camoratto AM, Jani JP, Angeles TS, Maroney AC, Sanders CY, Murakata C, Neff NT, Vaught JL, Isaacs JT, and Dionne CA. CEP-751 inhibits Trk receptor tyrosine kinase activity in vitro and exhibits anti-tumour activity. Int $\mathrm{J}$ Cancer. 1997; 72:673-679.

19. Behrens MM, Strasser U, and Choi DW. Gö 6976 is a potent inhibitor of neurotrophin-receptor intrinsic tyrosine kinase. J Neurochem. 1999; 72:919-924.

20. Wood ER, Kuyper L, Petrov KG, Hunter RN, and Harris PA. Discovery and in vitro evaluation of potent TrkA kinase inhibitors: oxindole and aza-oxindoles. Bioorg Med Chem Lett. 2004; 14:953-957.

21. Szul T, Grabski R, Lyons S, Morohashi Y, Shestopal S, Lowe M, and Sztul E. Dissecting the role of ARF guanine nucleotide exchange factor GBF1 in Golgi biogenesis and protein trafficking. J Cell Sci 2007;120:3929-3940.

22. Rios RM, and Bornens M. The Golgi apparatus at the cell centre. Curr. Opin. Cell Biol. 2003; 15:60-66.

23. Stauber T, Simpson JC, Pepperkok R, and Vernos I. A role for kinesin-2 in COPI-dependent recycling between the ER and the Golgi complex. Curr Biol. 2006; 16:2245-2251.

24. Tatu U, Braakman I, and Helenius A. Membrane glycoprotein folding, oligomerization and intracellular transport: effects of dithiothreitol in living cells. EMBO J. 1993; 12:2151-2157.

25. Lin ZP, Boller YC, Amer SM, Russell RL, Pacelli K A, Patierno SR, and Kennedy KA. Prevention of brefeldin A-induced resistance to teniposide by the proteasome inhibitor MG-132: involvement of NF-kappaB activation in drug resistance. Cancer Res. 1998; 58:3059-3065.

26. Sharpe LJ, Lu W, and Brown AJ. Akt phosphorylates Sec24: New clues into the regulation of ER-to-Golgi trafficking. Traffic. 2011; 12:19-27.

27. Lee MCS, Miller EA, Goldberg J, Orci L, and Schekman R. Bi-directional protein transport between the ER and Golgi.
Ann Rev Cell Biol. 2004; 20:87-123.

28. Rajagopal R, Chen ZY, Lee FS, and Chao MV. Transactivation of Trk neurotrophin receptors by G-protein coupled receptor ligands occurs on intracellular membranes. J Neurosci. 2004; 24:6650-6658.

29. Lee FS, Rajagopal R, Kim AH, Chang PC, and Chao M $\mathrm{V}$. Activation of Trk neurotrophin receptor signaling by pituitary adenylate cyclase-activating polypeptides. J Biol Chem. 2002; 277:9096-9102.

30. Lee FS, Rajagopal R, and Chao MV. Distinctive features of Trk neurotrophin receptor transactivation by G proteincoupled receptors. Cyto Growth Fact Rev. 2002; 13:11-17.

31. Miller FD, and Kaplan DR. On Trk retrograde signaling. Neuron. 2001; 32:767-770.

32. Bhattacharyya A, Watson FL, Pomeroy SL, Zhang Y, Stiles $\mathrm{CD}$, and Segal RA. High-resolution imaging demonstrates dynein based vesicular transport of activated Trk receptors. Int J Neurobiol. 2002; 51:302-312.

33. Moughamian AJ, Osborn GE, Lazurus JE, Maday S, and Holzbauer ELF. Ordered recruitment of dynactin to the microtubule plus-end is required for efficient initiation of retrograde axonal transport. J Neurosci. 2013; 33:1319013203.

34. Mayinger P. Signaling at the Golgi. Cold Spring Harb Perspect Biol. 2001; 3:a005314.

35. Ruggeri P, Farina AR, Di Ianni N, Cappabianca L, Ragone M, Ianni G, Gulino A, and Mackay AR. The TrkAIII oncoprotein inhibits mitochondrial free-radical ROS-induced death of SH-SY5Y neuroblastoma cells by augmenting SOD2 expression and activity at the mitochondria, within the context of a tumour stem cell-like phenotype. PLOS ONE. 2014; 9:e94568 